\title{
INDEX OF DISCUSSION REMARKS
}

(Numbers in italics refer to the Invited Reports)

Baranov, V. B. 266, 268

Bisnovatyi-Kogan, G. S. 236, 298, 299

Boyarchuk, A. A. 281, 299, 302, 304

Burke, B. 87, 89, 184, 190, 195, 196, 199, 265, $350,351,354,358$

Busemann, A. 375

Colgate, S. A. 19, 135-138, 140-143, 146-149, 217, 237-239, 245, 248

Csada, I. K. 195

Davis, L. 143, 184, 191, 266-268

Dolginov, A. Z. 322

Drobishevskii, E. M. 301

Dubov, E. E. 137

Dyson, J. 293, 351, 352

Field, G. B. 20, 21, 51, 78, 81, 83, 84, 89, 90, 93 , $95,96,102,104-106,140,141,148,149,184$, 189-192, 198, 199, 244, 248, 268, 295, 297, 298, $301,327-329,332,354,355,359,360,364$, $379-381$

Gershberg, R. E. 305

Goltsborthy, F. A. 94-96, 378

Gorbatskii, V. G. 300, 301

Gordon, I. M. 138, 269, 271, 304

Grebinskii, A. S. 194

Greenberg, J. M. 84, 195, 306, 322, 323, 325, 328-333

Habing, H. J. 84, 87, 88, 105, 303, 331, 379

Hulst, H. C. v. d. 3, 18-20, 86, 144, 185, 186, 192 , $217,271,304,322,324,326,329-335,362,364$

Kadomtsev, B. B. 108

Kaplan, S. A. 133, 135, 141, 142, 146, 374, 378

Kardashev, N. S. 193, 244

Karpman, V. I. 89, 147

Konyukov, M. V. 265, 266

Krat, V. A. 237, 300

Kurt, V. G. 322, 324, 325

Lüst, R. 107, 241, 249, 265-269, 378

Lynds, B. T. 328, 332, 381

Menon, T. K. 19, 87, 142, 190, 194, 245, 247, $333,347,349,356,358,359$
Mestel, L. 89, 107, 142, 187, 189, 198, 236, 248, 265, 295, 301, 364, 380

Meyer, F. 139, 263, 265, 269

Mezger, P. G. 93, 96, 336, 347-356

Mills, B. Y. 90, 189, 190, 246, 247

Nikolaev, Yu. M. 267

Ozernoi, L. M. 85, 193, 215-217, 247, 331, 353, 382

Parker, E. N. $83,84,89,106,140-146,149,168$, 185-187, 191, 194-199, 265-269, 295, 365

Pikel'ner, S. B. $18,82-84,88,89,102,106,142$, $148,149,185,187,193-199,236,244,291$, 293-295, 359-361, 379, 380

Podgornii, I. M. 144, 147

Pottasch, S. R. 91, 272, 294, 298, 303, 349, 355, 363

Rozhkovskii, D. A. 323

Salpeter, E. E. 221,320

Severnyi, A. B. $263,265,271$

Shklovskii, I. S. 19, 247, 304, 329, 333, 334, 349, 352, 363, 384

Shulman, L. M. 89, 326, 327

Silk, J. 78, 242, 294, 295, 361

Sorochenko, R. L. 350, 351, 356

Spiegel, E. A. 20, 21, 82, 83, 148, 149, 201, 215 , $217,269,381$

Stecher, T. P. 267, 294, 295, 298, 299, 316, 324 $326,329,330$

Sunyaev, R. A. 77, 78, 80, 105, 106, 238, 242, 244

Syrovat-skii, S. I. 18, 19, 84, 135-138, 141, 190 , 192, 238, 240, 245

Thomas, R. N. 20, 87, 88, 101, 104, 140, 266, 293, 294, 297, 299, 303, 304, 383

Toomre, J. 96, 383

Tsytovich, V. N. 108, 137-142, 187, 194, 238-241, 266

Varshalovich, D. A. 333, 334

Verschuur, G. L. 20, 85, 86, 89, 90, 102, 150, 189, 191-195, 198, 199, 245, 302, 323, 347-349, 358, 378,382 
Weaver, H. F. 20, 22, 85-90, 185, 186, 192, 333, Woltjer, L. 18, 19, 146, 185, 191-195, 229, 239349 $248,363,364$

Weliachew, L. 357

Weymann, R. J. 104, 191, 215, 248, 291, 294, 298, $299,330,361,379$

Zasov, A. V. 196

Woerden, H. v. 18, 19, 85, 86, 89, 95, 96, 98, 101, Zel'dovich, Ya. B. 81, 147, 193-196, 217, 241, $184,185,196,198,267,332,352,355-358,369$, 381,382 363

Zimmermann, H. 332 